\title{
Polymerization of 2-Oxabicyclo[2.2.2]octane
}

\author{
Takeo Saegusa, Tetsuo Hodaka, and Hiroyasu FujII \\ Department of Synthetic Chemistry, Kyoto University, \\ Sakyo-ku, Kyoto, Japan.
}

(Received April 1, 1971)

\section{KEY WORDS 2-Oxabicyclo[2.2.2]octane / Cationic Polymerization / Ring-opening Polymerization / Cyclohexane Ring / Six-membered Cyclic Ether / Conformation /}

Here we wish to report on the ring-opening polymerization of six-membered cyclic ether. Six-membered cyclic ether is lacking in ring strain and so far its polymerization has not been known to have been achieved. However, 2oxabicyclo[2.2.2]octane 1, one of the six-membered cyclic ethers, was polymerized in this study. Its polymerizability may be due to the ring strain derived from the cyclohexane ring having a boat form.

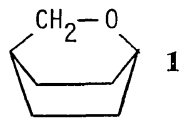

According to Bruice, et al., ${ }^{1} \mathbf{1}$ was prepared and purified by treating its methylene chloride solution with calcium hydride, followed by repeated sublimation.

The results of polymerization with some cationic catalysts are summarized in Table I.

The polymerization products of sticky solids were mostly soluble in methylene chloride and

Table I. Polymerization of 2-oxabicyclo[2.2.2] octane $\mathrm{o}^{\mathrm{a}}$

\begin{tabular}{lccccc}
\hline Catalyst & $\mathrm{mM}$ & Cocatalyst & $\mathrm{mM}$ & $\begin{array}{c}\text { Time, } \\
\mathrm{hr}\end{array}$ & $\begin{array}{c}\text { Yield, } \\
\%\end{array}$ \\
\hline $\mathrm{BF}_{3} \mathrm{THF}$ & 0.33 & Epichlorohydrin & 0.33 & 70 & 7.4 \\
$\mathrm{SnCl}_{4}$ & 0.33 & Epichlorohydrin & 0.33 & 24 & 27 \\
$\mathrm{SbCl}_{5}$ & 0.33 & Epichlorohydrin & 0.33 & 24 & $40^{\mathrm{b}}$ \\
$\mathrm{Et}_{3} \mathrm{OSbCl}_{6}$ & 0.11 & - & - & 40 & 3.9 \\
\hline
\end{tabular}

a Monomer, $6.6 \mathrm{mM} ; \overline{\mathrm{CH}}_{2} \overline{\mathrm{Cl}}_{2}, 1.5 \mathrm{ml} ; 0^{\circ} \mathrm{C}$.

b The molecular weight of the soluble part was 730 by VPO (in $\mathrm{CHCl}_{3}$ ). purified by precipitation in cold petroleum ether. The strong and broad absorption band at 1100 $\mathrm{cm}^{-1}$ in its IR spectrum shows the existence of $\mathrm{C}-\mathrm{O}-\mathrm{C}$ linkages in the product polymer. In the NMR spectrum of the polymer (Figure 1) the absorptions at $\tau 6.50$ and 6.63 (total $3 \mathrm{H}$ ) are assignable to three protons of the methine and methylene groups linked to oxygen, and those at $\tau 7.8-9.1(9 \mathrm{H})$ to the protons of the cyclohexane ring.

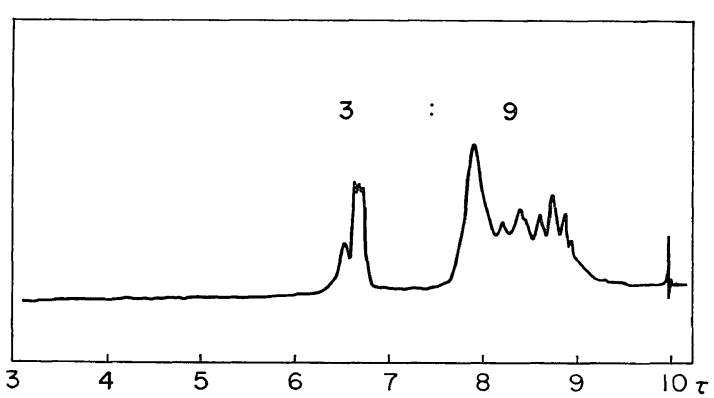

Figure 1. NMR spectrum of poly(2-oxabicyclo[2.2.2] octane): catalyst, $\mathrm{Et}_{3} \mathrm{OSbCl}_{6} ; 60 \mathrm{MHz}$; solvent, $\mathrm{CDCl}_{3}$.

Examination of the NMR spectrum $(\tau 7.8-9.1)$ of the product polymer has provided valuable information as to the stereochemistry of the propagation. The absorptions of the higher field

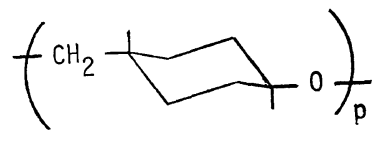

2 


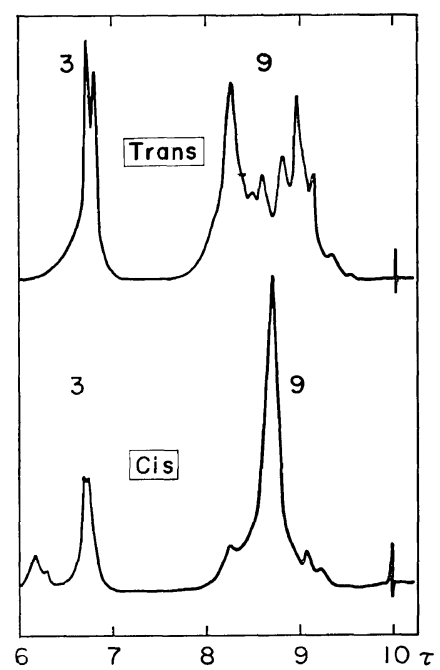

Figure 2. NMR spectra of trans- and cis-4-hydroxycyclohexyl carbinols: $\tau 6-10 ; 60 \mathrm{MHz}$; solvent, $\mathrm{CDCl}_{3}$. are generally attributed to the axial protons and those of the lower field to equatorial protons of the trans-1,4-disubstituted cyclohexane ring, which suggest the structure 2 .

Comparison of the NMR spectrum of this polymer with the spectra of cis- and trans-4hydroxycyclohexyl carbinols (glycol) (Figure 2) as reference compounds affords strong support for structure 2. The pattern of the spectrum of cyclohexane ring protons of trans-glycol is essentially the same as that of the polymer.

The formation of the polymer having a predominant trans-1,4-disubstituted cyclohexane ring structure leads to the following scheme of propagation.

On the basis of the bimolecular kinetics of cyclic ether polymerization, the back-side attack on the propagating oxonium by monomer 1 may involve the inversion of the $C^{1}$ configuration of the cyclohexane ring by the cleavage of
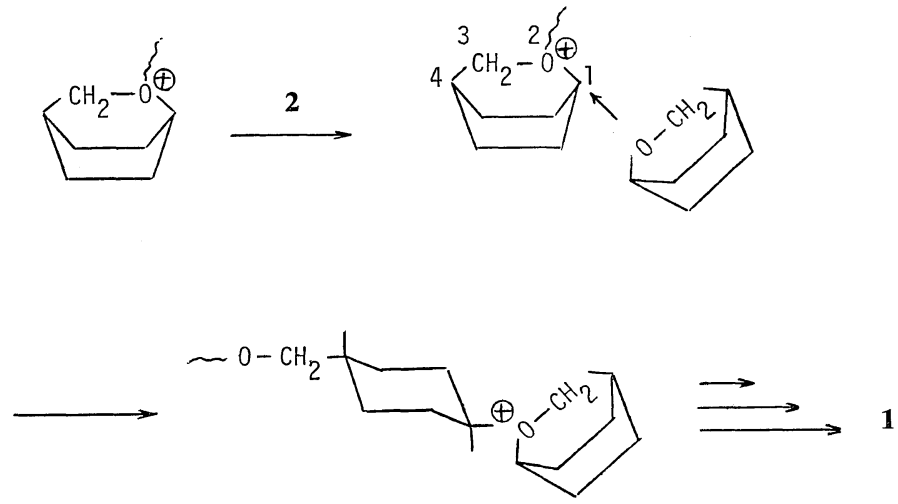

$\mathrm{C}^{1}-\mathrm{O}^{2}$ linkage. In the scheme, the more stable structure of equatorial methylene is given. This finding is taken to exclude the other possible means of attack at the bridge methylene carbon at position 3. In the case of the attack at $\mathrm{C}^{3}$ with the cleavage $\mathrm{C}^{3}-\mathrm{O}^{2}$ linkage, the cis configurations of the positions 1 and 4 should remain unchanged.
Further details will be published in a later paper.

\section{REFERENCE}

1. T. A. Giudici and T. C. Bruice, J. Org. Chem., 35, 2386 (1970). 